\title{
Morphogenesis of the $C$. elegans hermaphrodite uterus
}

\author{
Anna P. Newman ${ }^{1, \star}$, John G. White ${ }^{2, \dagger}$ and Paul W. Sternberg ${ }^{1}$ \\ ${ }^{1}$ Howard Hughes Medical Institute, Division of Biology 156-29, California Institute of Technology, Pasadena, CA 91125 , USA \\ ${ }^{2}$ MRC Laboratory of Molecular Biology, Hills Road, Cambridge, UK
}

*Author for correspondence at present address: Verna aned Marrs McLean Department of Biochemistry, Baylor College of Medicine, Houston, Texas 77030-3498, USA tPresent address: Laboratory of Molecular Biology and Department of Anatomy, University of Wisconsin, Madison, Wisconsin 53704, USA

\section{SUMMARY}

We have undertaken electron micrographic reconstruction of the Caenorhabditis elegans hermaphrodite uterus and determined the correspondence between cells defined by their lineage history and differentiated cell types. In this organ, many cells do not move during morphogenesis and the cell lineage may function to put cells where they are needed. Differentiated uterine cell types include the toroidal ut cells that make structural epithelium, and specialized utse and uv cells that make the connection between the uterus and the vulva. A cell fate decision in which the anchor cell $(\mathrm{AC})$ induces adjacent ventral uterine intermediate precursor cells to adopt the $\pi$ fate, rather than the ground state $\rho$, has profound consequences for terminal differentiation: all $\pi$ progeny are directly involved in making the uterine-vulval connection whereas all $\rho$ progeny contribute to ut toroids or the uterine-spermathecal valve. In addition to specifying certain uterine cell fates, the AC also induces the vulva. Its multiple inductions thereby function to coordinate the connection of an internal to an external epithelium. The AC induces the $\pi$ cells and ultimately fuses with a subset of their progeny. This is an example of reciprocal cell-cell interaction that can be studied at single cell resolution. The $\mathrm{AC}$ is thus a transitory cell type that plays a pivotal role in organizing the morphogenesis of the uterine-vulval connection.

Key words: Caenorhabditis elegans, hermaphrodite uterus, cell fate, morphogenesis, anchor cell

\section{INTRODUCTION}

Several general strategies may be used to specify fate during development. These include asymmetric segregation of determinants prior to cell division, induction, and identification of position within an overall coordinate system governing the body plan of the animal. Depending on the developmental strategies used, organs may be derived clonally, by multiple sublineages, or by a combination of the two. When cell-cell interactions are important, the communication may be bi-directional. For instance in the development of the vertebrate kidney, the uteric bud is required for the survival of the adjacent mesenchyme but also depends on the mesenchyme to develop and branch properly (Grobstein, 1955, 1956). While such cases of reciprocal induction have positive consequences for both cell types, it would also be possible for one side of the interaction to result in the responding cells losing their identity, either through cell death or cell fusion, both of which have important roles in development (Wakelom, 1988; Wylie et al., 1980; Yanagimachi, 1988). This might be a way to terminate the inducing capabilities of a cell once its job is done.

The nematode C. elegans is a self-fertilizing hermaphrodite. The entire gonadal lineages have been described (Kimble and Hirsh, 1979) so questions of fate specification during organogenesis can be studied with single cell resolution. The hermaphrodite gonad is bi-lobed, with germ line maturing in both arms (Hirsh et al., 1976). A distal tip cell at the end of each arm directs germ line proliferation, while maturation occurs as the germ cells move proximally towards the center of the gonad (Kimble and White, 1981). Oocytes, which are surrounded by sheath cells, pass through the oviduct, which has contractile activity, and are pushed into the spermatheca, where they are fertilized. Fertilized eggs mature in the uterus and are expelled through the vulva as a result of muscle contractions carried out by specialized uterine and vulval muscles.

How an internal and external epithelium become connected is a fundamental problem in developmental biology. In the $C$. elegans hermaphrodite gonad, the question is how the structural integrity of the animal is maintained while the uterus and vulva develop and become connected. Ablation experiments have demonstrated that a specialized cell of the ventral uterus, the anchor cell (AC) plays a key role. Early in uterine development, mutual signaling between two initally equivalent cells results in one cell adopting the $\mathrm{AC}$ fate while the other becomes a ventral uterine precursor (VU) cell (Kimble, 1981; Seydoux and Greenwald, 1989). This process is mediated by LIN-12, a founding member of the Notch family of receptors, and likely by LAG-2, a molecule homologous to the Notch ligands Delta and Serrate (Greenwald et al., 1983; Henderson et al., 1994; Tax et al., 1994; Wilkinson et al., 1994; Yochem et al., 1988). Subsequently, the AC induces the vulval precursor cells (VPCs) that will produce the vulva (Kimble, 1981; Sternberg and Horvitz, 1986) via the lin-3-encoded growth factor (Hill and Sternberg, 1992; Katz et al., 1995) and the let-23-encoded tyrosine kinase receptor (Aroian et al., 1990). Finally, the AC induces adjacent VU granddaughters 
(intermediate precursor cells) to adopt the $\pi$ fate, rather than the ground state $\rho$, using the lin-12-encoded receptor (Newman et al., 1995). $\pi$ cells are required for egg-laying and differ from $\rho$ cells in the number of progeny produced, axes of division, and morphology of their progeny. An attractive hypothesis was that the AC might physically bring together the vulval and uterine cells it induces to make the final specialized egg-laying apparatus. However, the final fate of the AC was not known.

While the entire lineage of the $C$. elegans hermaphrodite gonad has been determined, terminal cell types have not been defined. Here we have used electron micrographic (EM) reconstruction to describe the nature and number of cells in the uterus. We have also followed the positions of all nuclei from the end of the lineage through the formation of the mature structure, thus correlating differentiated fate with lineal position. These data suggest the developmental strategies used to make the $C$. elegans uterus. Specifically, we found that lineage bifurcations do not always correlate with differentiated fate. For the epithelial toroidal cells, our data are consistent with the lineage being used to put cells in the right place. Finally, we have followed the AC late in development and determined that it loses its distinct identity when it fuses with a subset of the $\pi$ progeny. Induction of the $\pi$ fate by the AC and the subsequent fusion of the AC with $\pi$ progeny constitutes a striking example of reciprocal cell-cell interactions.

\section{MATERIALS AND METHODS}

The wild-type N2 strain was cultured using standard procedures (Brenner, 1974). Cell lineages were followed using Nomarski optics (Sulston and Horvitz, 1977). The data on morphogenesis in intact N2 animals is derived from 42 animals that were followed for all or part of the L4 larval period. Nomarski photomicrographs were taken with a Zeiss standard 16 with microflash on Kodak Techpan film. Cells were ablated with a laser microbeam (Avery and Horvitz, 1987; Sulston and White, 1980). Criteria for distinguishing $\pi$ and $\rho$ fates are discussed in detail by Newman et al. (1995). The vulF cells, which are the most dorsal of the mature vulval cells (White, 1988), are the progeny of the inner granddaughters of P6.p (Sulston and Horvitz, 1977). The vulE cells are the next most dorsal mature cells and derive from the outer P6.p granddaughters. Serial EM reconstructions were performed as described (White et al., 1986). Two animals in the mid L4 stage and one in the late L4 were reconstructed. The late L4 uterus seems to be completely formed, being almost identical in structure and organization to that of the adult apart from the presence of eggs (J. G. White and E. Southgate, unpublished observations). We used the MH27 antibody (Waterston, 1988) to stain adherens junctions using described modifications (Evans et al., 1994) of a freeze-cracking technique (Albertson, 1984; Strome, 1983). Preparations were examined on an MRC-600 confocal microscope.

$\pi$ progeny nuclei were followed on either the left or the right side of the animal from the start of their migrations during early to midL4 through the beginning of L4 lethargus. In 16/16 animals, the nuclei of both ventral outer $\pi$ progeny (VT4 and VT8) remained proximal to the vulva, while those of VT5, 6, 7, and 9 migrated, reaching ut2 either anteriorly or posteriorly. During morphogenesis, the AC nucleus moves from its medial position to either the left or the right of the animal and joins the migrating $\pi$ progeny nuclei. AC migration was followed in those ten animals in which it moved to the side being followed; in 10/10, it reached ut2. The 16 animals followed displayed a bias of movement towards the posterior, both for the migrating $\pi$ progeny nuclei (VT5, 6, 7, and 9) and the AC. In five animals, two of the four $\pi$ cell progeny nuclei migrated posteriorly; in three of four of these animals, the AC nucleus moved posteriorly. In 10 animals, three of the four $\pi$ cell progeny nuclei migrated posteriorly; in five of six of these animals, the AC nucleus migrated posteriorly. In the remaining animal, all four $\pi$ cell progeny nuclei migrated posteriorly.

\section{RESULTS}

\section{Structure of the hermaphrodite uterus}

We reconstructed the hermaphrodite gonad from electron micrographs of serial sections in the mid and late L4 stage. This structure has two two-fold axes of symmetry, anterior-posterior $180^{\circ}$ rotational symmetry about a dorsal-ventral axis centered at the vulva and mirror symmetry around the longitudinal axis of the gonad (Fig. 1). There are 8 distinct regions in the somatic gonad: the connection to the vulva, the connection to the seam cells of the lateral epidermis, the uterus, the spermathecal valve, the spermatheca, the oviduct, the ovary sheath and the distal tip (Figs1-3). Here we focus on the structure of the uterus. Starting at the vulva there are three mononucleate cells (uv1-3; uterinevulval) on both the left and right side of the animal that form the connection of the uterus to the vulva; there is also a single dorsal uterine cell $(\mathrm{du})$ opposite the ventral vulval/uterine junction in this region (see Fig. 7C). The uterus is attached to specialized lateral epithelial cells (seam cells) by a single ' $\mathrm{H}$ ' shaped cell containing 9 nuclei (utse: uterine-seam cell). The two sides of the " $\mathrm{H}$ " run along the seam on each side of the hermaphrodite, attaching the uterus to this lateral epidermis. The central portion crosses the animal transversely, passing above the vulva and forming a thin laminar process between the uterus and the vulva. The utse has a major role in holding the uterus in the body and presumably functions to stop it from prolapsing. Next there is a sequence of four multinucleate toroidal uterine cells (ut1-4); these are followed by a toroidal spermathecal valve cell (also referred to as the spermathecal-uterine junction) containing four nuclei which has a ring of microfilaments that wrap around the inside face of the torus and presumably acts as a sphincter to close the valve in the adult (Kimble and Hirsh, 1979, White, 1988). The lumen of the valve cell is blocked by the junctional core, a bi-nucleate plug cell, in the L4; presumably this cell gets expelled from the gonad when eggs pass through the spermathecal valve. Distal to the spermathecal valve are the cells of the spermatheca, which is organized as an extended tube, and oviduct, a highly motile structure that seems to be used for picking an oocyte from the syncytial mass of germ cells and delivering it to the spermatheca where it is fertilized. The gonad no longer has a continuous sheath of somatic cells beyond this region. It is, however, partially enclosed by the sheath cells and completely surrounded by a basal lamina. The distal tip cell, as its name implies, is situated at the distal extremity of each arm of the gonad.

\section{Summary of uterine development}

The uterus comprises 60 of 84 of the descendants of two dorsal uterine precursor (DU) and three ventral uterine precursor (VU) cells (Kimble and Hirsh, 1979). The DU cells produce a total of 48 descendants ( 24 each), with 28 making uterine tissue, 12 contributing to the anterior or posterior spermatheca, and eight producing part of the uterine-spermathecal junction, including the junctional core (Fig. 4). The VU cells make a total of 36 progeny, with 32 contributing to the uterus and four to the 


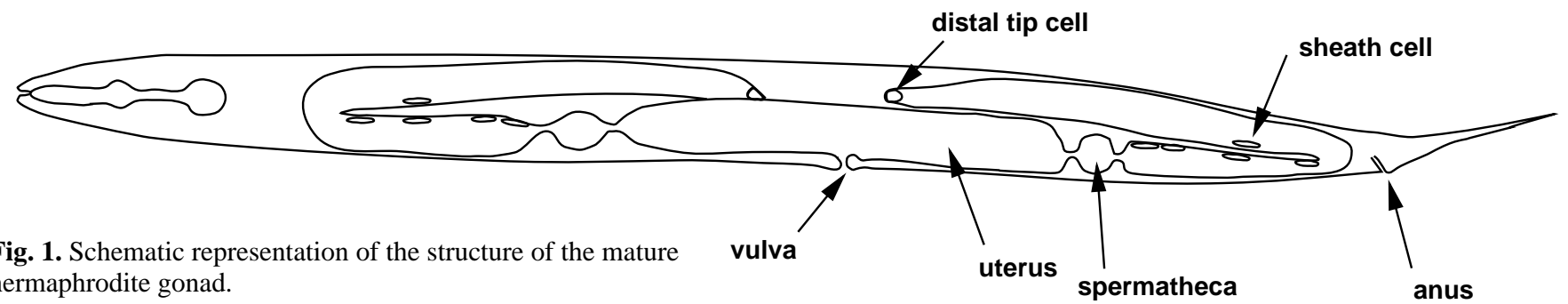

hermaphrodite gonad.

spermatheca

anus

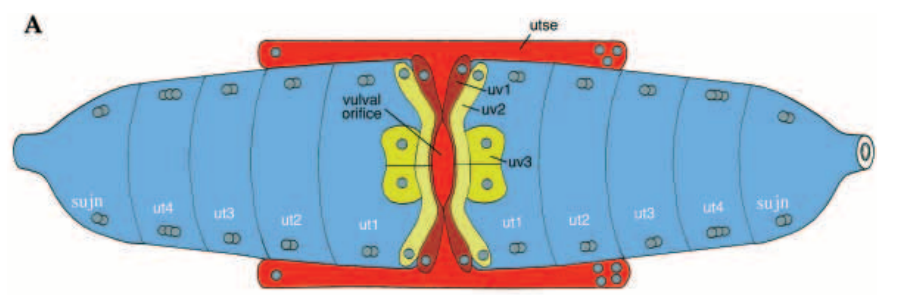

B

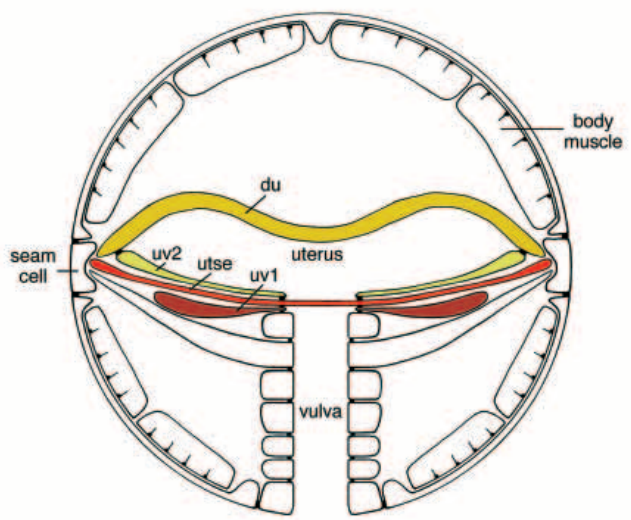

Fig. 2. Schematic representation of mature uterine structures.

General cell types are color coded: blue for ut toroids and uterinespermathecal junction, yellows for the du, uv2, and uv3 cells, and reds for uv1 and the utse, which make the connection to the vulva. (A) Ventral view depicting ut toroids, utse, uv cells, and spermathecal-uterine valve (i.e. junction: sujn). (B) Transverse view of the uterus at the center of the animal where it sits over the vulva. The uv1 cells are directly dorsal to the vulva, and the utse and uv2 are dorsal to it. The du cell forms the dorsal-most uterus in the center of the animal where no toroids exist. The lateral attachment of the utse to the seam is also shown.

uterine-spermathecal junction. Many cells fuse, producing the adult cell types.

The DU cells undergo five rounds of cell division; the VU cells undergo four. We have assigned names to the DU and VU great grandprogeny: DE1-8 (Dorsal Eight) and VT1-12 (Ventral Twelve; Table 1). The latter are the daughters of the VU-lineage intermediate precursor cells, which can be of either $\pi$ or $\rho$ fate. $\pi$ and $\rho$ cells differ in their division axes, number of progeny produced (Newman et al., 1995), and the type of progeny generated (this study).

By following the positions of nuclei from completion of the uterine lineages in early L4 through completion of morphogenesis late in L4 and correlating them with the data from EM reconstructions, we determined which cells defined by lineage make which structures. In the following sections, we describe these assignments for each cell type, detailing the migrations,

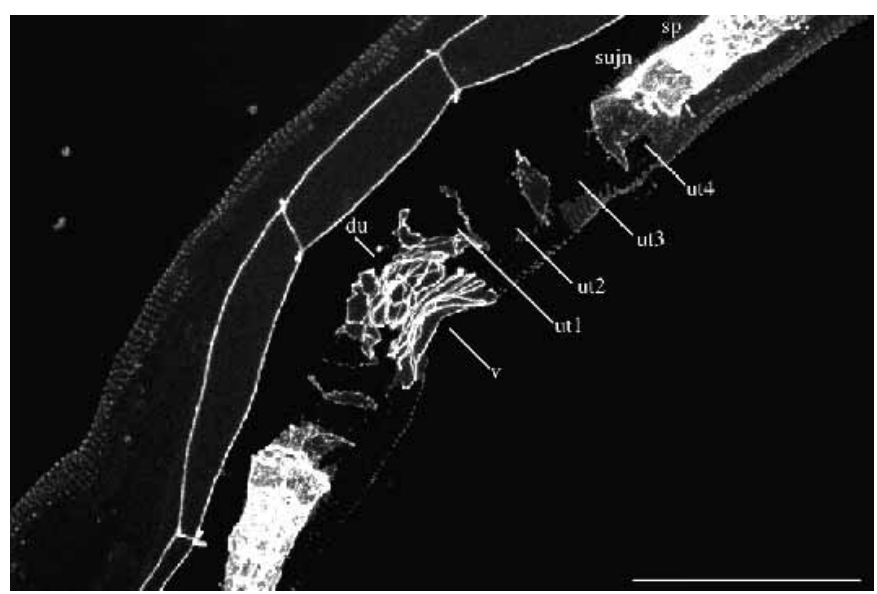

Fig. 3. Extended focus confocal image of the vulva, uterus, spermathecae, and uterine-spermathecal junctions of an L4 hermaphrodite animal visualized by staining with MH27 antibody, which delineates apical junctions. ut, ut toroid; v, vulva; sujn, spermathecal-uterine valve (junction); du, du cell; sp, spermatheca. Bar, $30 \mu \mathrm{m}$.

cell fusions and shape changes the uterine cells undergo during the process of differentiation. All descriptions below are per lateral side; numbers need to be doubled for the total number of nuclei per anterior-posterior position.

\section{Formation of the ut toroids}

Following the final uterine divisions during the beginning of the L4 (Kimble and Hirsh, 1979; see Figs 4, 5A,B), the uterus largely consists of two epithelial rows, ventral and dorsal (Fig. 6A). The exceptions to this are the most distal DU descendants (DE1, DE2, DE7, DE8) that have already separated from the uterus to form part of the spermatheca and the core cells of the spermathecal valves (Kimble and Hirsh, 1979), the $\pi$ cell progeny (VT4-VT9) that are lateral to the other uterine cells, and DE4, 5, whose daughters make some of the uv cells and the du cell that is dorsal to the vulva (see below). Thus, the two epithelial rows contain all the DU-derived nuclei except for the most proximal and most distal cells and all of the $\rho$ cell progeny.

The nuclei in the two epithelial rows comprise the ut toroids and the spermathecal valve (Fig. 5C). These nuclei do not undergo any appreciable movements during the L4. Instead, they remain approximately where they are born and maintain their positions along the anterior-posterior axis set up by the last round of cell division. This gives one dorsal and one ventral nucleus per anterior-posterior position, except that since VT2 has divided such that one of its daughters is under- 

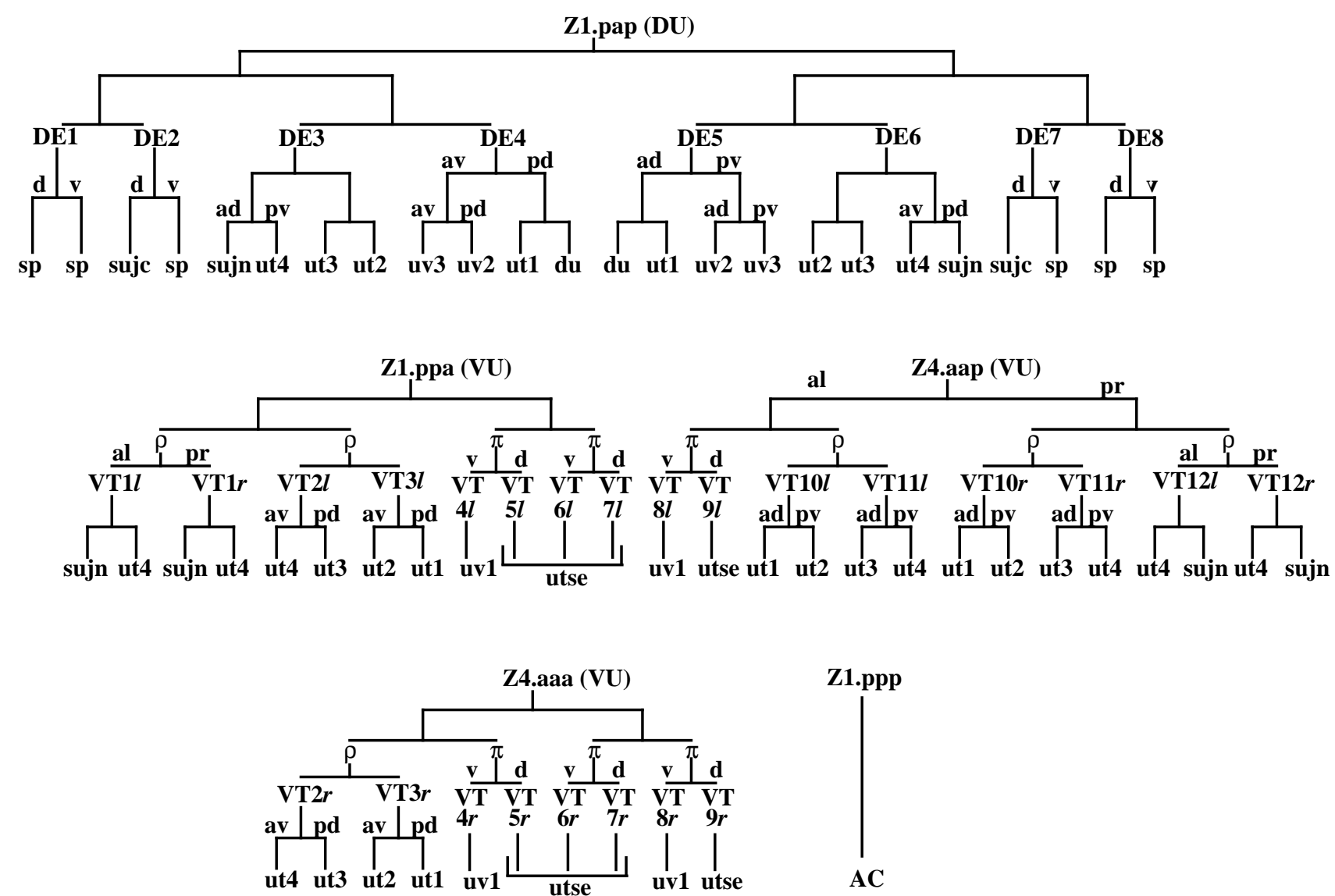

Fig. 4. Uterine lineages. Lineage diagrams depicting the differentiated progeny produced by the DU cell Z1.pap and the VU cells Z1.ppa, Z1.ppp, Z4.aap, and Z4.aaa when the 5R conformation is adopted. The pattern of cell divisions has been previously described (Kimble and Hirsh, 1979). The lineages of the two DU cells Z1.pap and Z4.apa are symmetric. See Kimble and Hirsh (1979) for a description of the 5R and 5L conformations and Newman et al. (1995) for $\pi$ and $\rho$ fates. Anterior is to the left. Cell divisions are anterior-posterior unless otherwise indicated. a, anterior; p, posterior; d, dorsal; v, ventral; 1, left; r, right; sp, spermatheca; sujn, spermathecal-uterine valve; sujc, spermathecaluterine valve core; ut, ut toroid; du, du cell. For convenience, we have named some of the intermediate cells DE1-DE8 (Dorsal Eight), VT1VT12 (Ventral Twelve).

neath one daughter each of VT1 and DE3a, there are three nuclei in that column. These nuclei comprise the ut 4 toroid, which has three nuclei per side; other toroids consist of one dorsal and one ventral nucleus per side. Thus, the differing numbers of nuclei in the ut4 versus other toroids are established during cell division and are not altered by cell migration.

Each toroid is formed when the four or six cells (including left and right) at that anterior-posterior position fuse. This conclusion is based on EM reconstructions of animals before, after, or in the process of fusion, and on antibody staining of the adherens junctions between the toroids (Fig. 3). EM reconstruction of an animal in mid-L4 revealed that some but not all of the ut fusions had occurred. For instance, the dorsal and ventral cells that will form ut 1 were fused on one side of the animal but not on the other. Thus, the fusions appear to occur during mid-L4 relatively early in morphogenesis. During morphogenesis, the cytoplasm of the toroids becomes thinner as the lumen forms, then enlarges, with the toroids surrounding it (Fig. 6D,F).

While all ut toroids are formed by fusion of DU and VU descendants, there may be differences in their subsequent differentiation. For instance, ut 1 is smaller than ut 2 and ut3. The ut 2 and ut 3 nuclei characteristically enlarge beginning relatively early in morphogenesis (Fig. 6B). Moreover, the sper- mathecal valves, which, like the toroids, result from the fusion of DU and VU descendants are nonetheless highly specialized cell types. The valve cells adhere closely to the 'core' of the valve (Kimble and Hirsh, 1979) and later have a distinctive morphology consisting of rings of filaments that enable them to contract and function as sphincters (White, 1988).

\section{Fusion of a subset of $\pi$ progeny and the AC to form the utse}

The $\pi$ cells divide asymmetrically during L3 lethargus to produce larger dorsal and smaller ventral daughters (Fig. 5A). Following this, the $\pi$ progeny can be divided into two classes: four (on each side) contribute to the utse, while two become uv1 cells (see below). Interestingly, although the $\pi$ cell divisions are unequal, the fate of the differentiated progeny is determined at least partially independently: the two ventral outer progeny (VT4, VT8) become uv1, while all of the dorsal progeny plus the inner ventral daughter (VT5, 6, 7, 9) contribute to the utse.

By Nomarski optics, the $\pi$ progeny that contribute to the utse can be easily distinguished from those that become uv1 cells since the nuclei of the former undergo long-range migrations whereas those of the latter remain closely associated with the vulva (Fig. 5, compare B and C). The first stage in these migra- 
Table 1. Uterine and vulval cell nomenclature

\begin{tabular}{|c|c|}
\hline Abbreviation* & Cell type \\
\hline $\mathrm{AC}$ & Anchor cell \\
\hline VU cell & Ventral Uterine precursor cell \\
\hline DU cell & Dorsal Uterine precursor cell \\
\hline$\pi$ cell & $\bar{V} U$-lineage intermediate precursor cell (i.e. VU cell granddaughter) of fate $\pi$ (induced fate) \\
\hline$\rho$ cell & VU-lineage intermediate precursor cell (i.e. VU cell granddaughter) of fate $\rho$ (uninduced fate) \\
\hline VT1-12 & Ventral Twelve 1-12 \\
\hline & 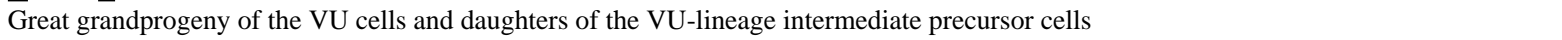 \\
\hline DE1-8 & Dorsal Eight $1-8$ \\
\hline & $\overline{\text { Great grandprogeny of the DU cells }}$ \\
\hline ut cells & uterine toroidal epithelial cells that constitute the bulk of the uterus; formed by fusion of four (ut1-3) or six (ut4) cells \\
\hline du cell & dorsal uterine cell; cell forming dorsal epithelium in central region of the uterus; formed by fusion of four cells \\
\hline uv cells & uterine-vulval cells; mononucleate interfacial cells between the uterus and vulva \\
\hline utse & $\begin{array}{l}\text { uterine seam cell; membranous cell formed by fusion of eight } \pi \text { cell progeny and the AC; attaches the uterus to the lateral epidermis (seam) } \\
\text { and forms a thin laminar process dorsal to the vulva }\end{array}$ \\
\hline VPC & Vulval Precursor $\underline{\text { Cell; }}$ epidermal cell with potential to generate vulval tissue \\
\hline vulF cells & vulval F cells. The most dorsal of the mature vulval cells and progeny of the inner granddaughters of one of the VPCs (P6.p). \\
\hline vulE cells & vulval E cells. The next most dorsal of the mature vulval cells and progeny of the outer granddaughters of one of the VPCs (P6.p) \\
\hline
\end{tabular}

*Upper-case letters refer to precursor cells or intermediate precursor cells, lower-case letters refer to differentiated cell types, Greek names refer to cell fates.

tions involves short-range rearrangements that are variable; however, in essence, VT6 moves dorsally and some of the nuclei begin to move distally from their initial location (Fig. 6G). Generally, these minor movements are all that occur during the first four hours of L4, during which time the AC remains dorsal to the vulva. By mid-L4, major changes are occuring in the uterus. A fluid-filled lumen is beginning to form between the dorsal and ventral layers of the uterus that also extends above the AC. The AC becomes less round and ruffled as the tissue dorsal to the vulva thins out and its nucleus begins to resemble those of the migrating $\pi$ progeny. The AC nucleus begins to migrate anteriorly or posteriorly (Fig. $6 \mathrm{H}$ ), and also moves from its medial position to join these $\pi$ progeny nuclei, which are either left or right lateral. EM reconstructions indicate that the $\mathrm{AC}$ fuses with the utse prior to the time of extensive nuclear migrations (Fig. 7, compare A and B) and appears to be engulfed by it. In a mid-L4 animal in which the AC was still dorsal to the vulva, it had fused with the two nascent utse cells on either side. All the cells that form the utse in this animal had fused. A reasonable interpretation of our observations is that a subset of $\pi$ progeny fuse to form the utse, which then fuses with the AC. Dorsal to the vulva, the utse is a thin laminar process. It faces both the uterine and the vulval lumens and is an unusual cell in that it has two apical surfaces.

Longitudinally, the utse extends to ut 2 on both sides (Fig. 2A). In a mid-L4 animal, the cytoplasm was fully extended. However, the utse nuclei, which eventually migrate approximately to ut2 (see below), were not yet there. Thus, the utse appears to first extend a cell process that then attaches to the seam. This lateral attachment may cause the utse nuclei to be squeezed out to the ends.

We have followed the migrations of the utse nuclei on one side each of 16 animals using Nomarski optics. There seems to be a bias toward the posterior, both for the $\pi$ progeny and

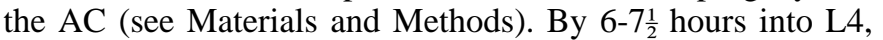
all migrating utse nuclei have reached their final location: at or slightly past VT3a or VT10p (ut2 nuclei; Fig. 5C). By about eight hours into L4, a thin line forms slightly ventral to the seam, extending from the anterior to the posterior utse nuclei, containing $\pi$ progeny and AC nuclei at its corners (Figs 5D,
$6 \mathrm{I}, \mathrm{J})$. This line has previously been hypothesized to represent attachment of the utse to the seam (E. Hedgecock, personal communication).

EM reconstructions have provided further insight into the nature of the uterine attachment at the seam. Specifically, the basal lamina that surrounds the uterus becomes thicker in the vicinity of the utse. This induces shape changes in the seam cells (and likely the production of filaments) such that they attach to the cuticle (Fig. 7C). The basal lamina encases other uterine cells that are attached to the seam, for example, the du cell in Fig. 7C.

We previously proposed that eight of the twelve $\pi$ progeny nuclei make utse since mutant animals that fail to specify $\pi$ cells also make no utse, while those with excess $\pi$ cells have many additional nuclei in the utse (Newman et al., 1995). Our direct observations of $\pi$ progeny movements strongly reinforce this conclusion and indicate that the utse is formed by fusion of the AC with the eight nuclei consisting of VT5, 6, 7 and 9 on the left and right.

\section{$\pi$ cells are required to make the thin uterine-vulval connection and for late differentiation of the AC}

To evaluate directly whether $\pi$ progeny make the thin planar process of the utse that separates the uterus and vulva, and whether these cells are required for the late fate of the AC, we ablated the $\pi$ cells during the L3 and followed animals by Nomarski optics from early to mid-L4 through the beginning of L4 lethargus. In 8/8 animals, the AC failed to migrate and its final position was either directly dorsal or immediately anterior or posterior to the vulva. Often, the cytoplasm surrounding the $\mathrm{AC}$ was thick, giving the AC a bloated appearance. The characteristic thinning of the tissue dorsal to the vulva did not occur. These results indicate that the utse is necessary for the AC to change its morphology and migrate, consistent with the EM observation that the utse fuses with the AC.

\section{The ventral outer $\pi$ progeny make uv1 cells}

When the $\pi$ cells divide during L3 lethargus, the ventral outer progeny (VT4, V8) are born near the most dorsal vulval cells, vulF. VT4 and VT8 remain in close association with these cells 

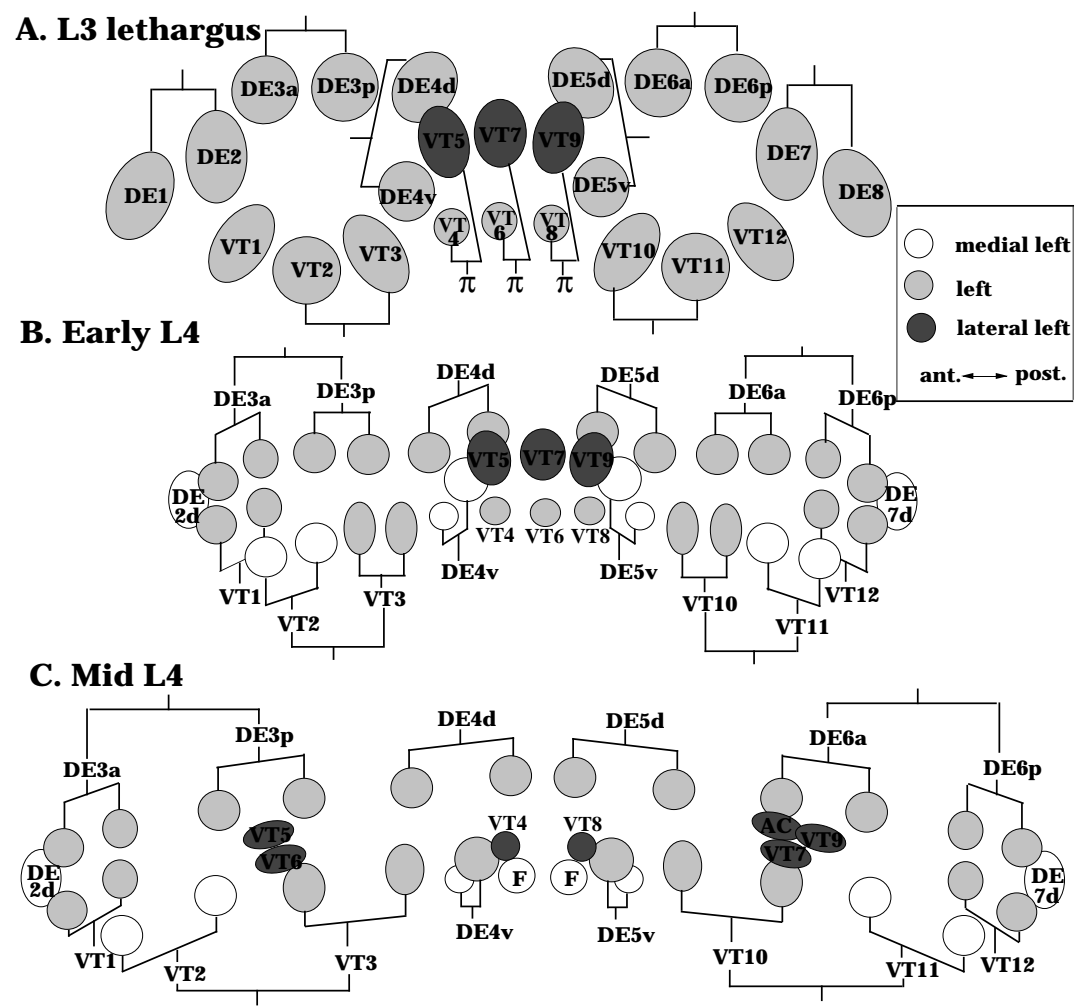

Fig. 5. Schematic representation of nuclear positions during uterine morphogenesis. The nuclei on the left side of the animal are depicted; both sides are symmetric. (A) Configuration of nuclei during L3 lethargus when ventral uterine cells have completed the third round of division and some dorsal uterine cells have undergone the fourth round. Cells are named after the third round of division. The division of DE4 and DE5 has led to the delamination of D4v and D5v from the dorsal epithelium. (B) During the early L4, the basic plan of the uterus consists of two epithelial rows, which will not change much during morphogenesis. Specialized cells will undergo migrations and local rearrangements; these include the $\pi$ progeny, VT4VT9, and the daughters of DE4v and DE5v. DE2v and DE7 $\mathrm{v}$ and the progeny of DE1 and DE8 contribute to the spermatheca; these cells are not shown in B or C. (C) By mid-L4, the dorsal daughters of DE4v and DE5v have become more ventral and interposed between VT4 or VT8 and the ventral daughters of $\mathrm{DE} 4 \mathrm{v}$ and DE5v so that the characteristic association between (to the anterior) vulF, VT4, DE4vd, and DE4vv is established. During mid to late L4, the VT5, VT6, VT7, VT9 and AC nuclei are migrating to their final positions at, or slightly past, VT3a and VT10p. (D) By L4 lethargus, local rearrangements have occurred in the region proximal to the vulva so that while (to the anterior) DE4vd and VT4 still remain closely associated with one another and the vulva, they are more ventral and closer to vulE than vulF, while DE4vv is somewhat more distal. A thin line has formed just below the seam with the migrating $\pi$ progeny and AC nuclei at its corners. There is variability in the positions of the VT5-9 and AC nuclei (see Results) and in the final relative positions of the VT4 and VT8 nuclei with respect to those of DE4vd and DE5vd: VT4 and VT8 are generally slightly more distal than DE4vd or DE5vd (5/8), as depicted in D, but are sometimes more proximal (1/8) or at the same anterior-posterior position (2/8). The connections of the corresponding cells were not variable in the three animals reconstructed. Nuclei in the lower part of $\mathrm{C}$ are color coded to correspond to the cell types shown in Fig. 2: blue, ut toroids; yellow, uterine uv2, uv3, and du cells; red, utse and uv1 (those uv cells contacting the vulva); sujn, spermathecal-uterine valve; sujc, spermathecal-uterine valve core. E: vulE cell; F: vulF cell.

for about the first 3/4 of L4, moving slightly but always remaining close enough to enable their corners to remain touching (Figs 5C, $6 \mathrm{G})$. EM reconstructions show that, beginning in mid-L4, only the uv1 cells are immediately dorsal to vulF. Thus, the ventral outer $\pi$ progeny make uv1. These cells have lateral nuclei and extend thin processes that contact the vulF cells (Fig. 7B). The thin process of the utse is dorsal to that of the uv1 cells, with which it makes adherens junctions. Since the utse forms the planar cell process that constitutes the interface between the uterus and the vulva, the two types of differentiated progeny made by $\pi$ cells (uv1 and utse) are those that contact the vulva.

\section{The most proximal DU cells make the uv2, uv3 and du cells that are dorsal to the vulva}

The toroids that make the body wall of the uterus do not extend proximally to the region dorsal to the vulva. A toroid in such a position might preclude formation of the thin uterine-vulval connection made by the utse. Instead, the mononucleate uv2 


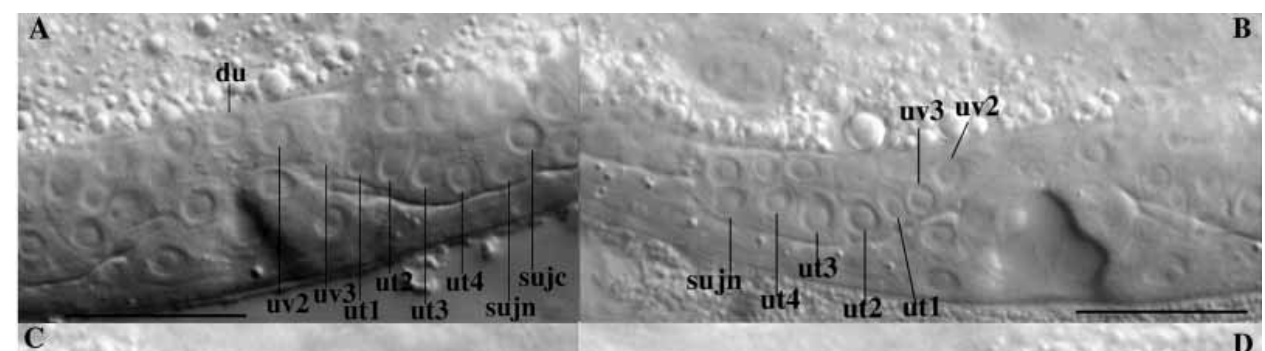

Fig. 6. Nomarski photomicrographs of the uterus during morphogenesis. Right (A,I,J) and left (B-H) lateral views. (A) Two rows of uterine epithelial cells early in L4. DE4v has divided to produce uv2 and uv3; uv2 (DE4vd) is still relatively dorsal. The ventral nucleus of the anterior ut toroids and spermathecal-uterine valve (sujn) are indicated, as are the anterior du cell (du),

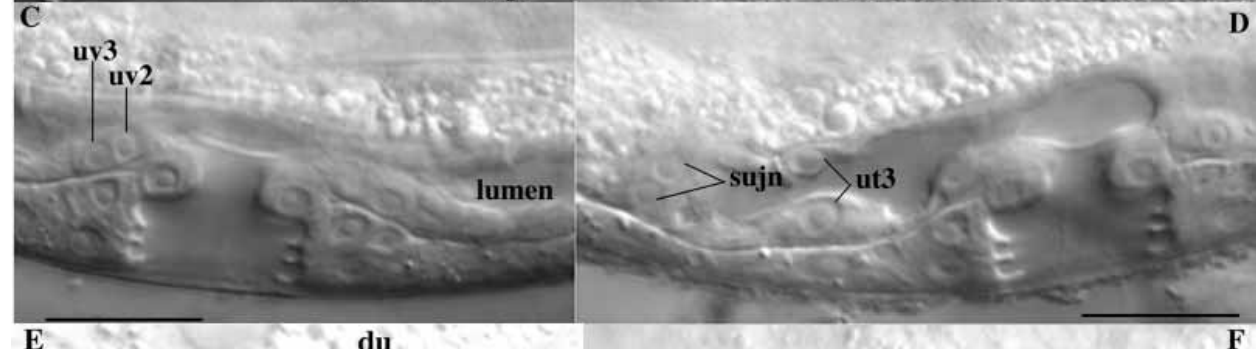
uv2, uv3, and valve core nuclei (sujc). (B) Same as A except that nuclei have begun to change in size so that, for example, the ventral ut 2 and ut 3 nuclei are particularly enlarged. (C) Mid-L4. The progeny of DE4v (uv2 and uv3) have become more ventral during morphogenesis and lumen formation. (D) Mid-L4. The cytoplasm surrounding the dorsal ut3 nucleus has

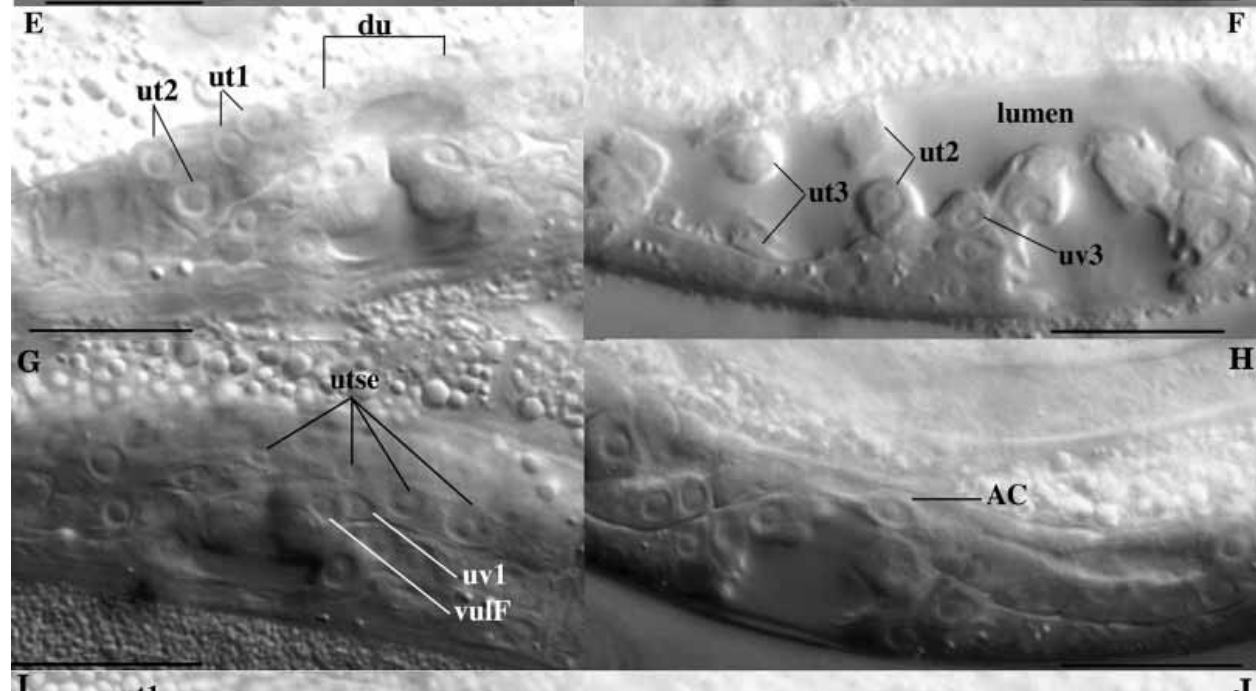
begun to thin during lumen formation. (E) Positions of ut1, ut 2 and du nuclei during mid- to late-L4. (F) Mid- to late-L4. The cytoplasm of the ut 2 and ut 3 cells has thinned so that just the portion of the cell containing the nuclei protrudes into the lumen. The uv3 cell can also be seen protruding into the uterine lumen. $(\mathrm{G}) \pi$ cell progeny during mid-L4. VT4 and VT8 (uv1) reside just dorsal to vulF. Of the utse nuclei, VT6 remains dorsal to the center of the vulva, while VT5, 7, 9 have begun their migrations to the posterior. $(\mathrm{H}) \mathrm{Mid}-$ $\mathrm{L} 4$. The $\mathrm{AC}$ has begun moving from its central position dorsal to the vulva towards the posterior. (I) L4 lethargus. Nuclei visible in a lateral plane, including those of the uv1, uv2, ut 1 and du cells. Two of the three posterior utse nuclei are visible, as is

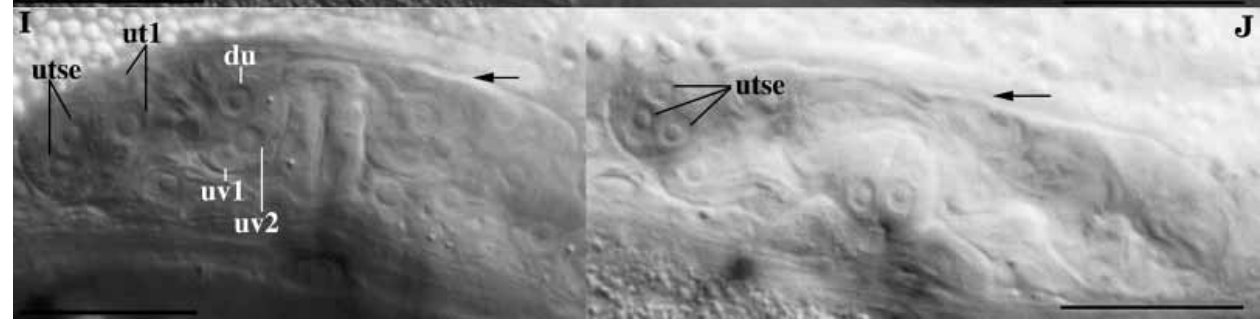

the thin line that curves around them (arrow). The ut 2 nuclei are at the same anterior-posterior position as the utse nuclei, but out of the plane of focus. (J) L4 lethargus, lateral plane containing utse nuclei. Nuclei of VT5, VT6, and the AC have migrated to ut 2 and a thin line (arrow) has formed with them at its corner. Bar, $20 \mu \mathrm{m}$.

and uv3 cells form the ventral epithelium, while the du cell (possessing four nuclei) makes the dorsal epithelium, in that region (Fig. 2B). We refer to these cells collectively as proximal epithelium. All are made by DE4 and DE5. These cells divide dorsal-ventrally to produce DE4d and DE5d, which remain part of the dorsal epithelium and DE4v and DE5v, which delaminate from this epithelium (Fig. 5A). All of these cells divide again and the more proximal daughters of DE4d and DE5d fuse to form the du cell (Figs 5C, 6E). The daughters of DE4v and DE5v become more ventral during morphogenesis (Fig. 6; compare $\mathrm{A}$ and $\mathrm{B}$ with $\mathrm{C}$ ). The DE4v and DE5v daughters make the uv2 and uv3 cells, DE4vv and DE5vv making uv3 and DE4vd and DE5vd making uv2. uv2, like uv1, eventually extends a thin process. However, in the case of uv2, this process contacts the uterine rather than the vulval lumen (Fig. 7B,C). Also, in contrast to uv1, the uv2 nucleus is first medial and then appears to become more lateral during stretching of the cell
(Fig. 6I). The utse process is ventral to that of uv2, so that in the region of the vulva, there is a 'sandwich' of three cytoplasmic processes, with uv1 being most ventral and contacting vulF, utse residing on top of it and forming a thin planar process between the uterus and vulva, and uv2 being most dorsal and contacting the uterine lumen (Fig. 7C). These cells make an extensive network of adherens junctions with one another.

In contrast to the other uv cells, uv3 does not extend a cytoplasmic process during the L4; rather, it remains compact and distinct (Fig. 6F). Of the uv cells, it is the most distal from the vulva. However, we have not found cells of this shape in the adult, possibly due to subsequent shape changes.

\section{DISCUSSION}

We have described the structure and development of the $C$. elegans uterus and defined the component cell types. The 
toroidal ut cells constitute the bulk of the uterus. The membranous utse attaches the uterus to the lateral body wall and forms a thin laminar process dorsal to the vulva. The mononucleate uv cells are interfacial between the uterus and the vulva. The du cell forms the uterine cap dorsal to the vulva in the region where there are no toroids. Distinct developmental strategies seem to be used in the specification of the different cell types, as discussed below. Uterine development highlights the role of cell fusions during morphogenesis.

\section{Formation of ut toroids and proximal epithelium}

The ut toroids are made by fusion of cells that undergo no migrations during uterine morphogenesis: the cell lineage puts cells in location appropriate for function. The cells are born such that they form two rows of cells with a dorsal uterine precursor (DU) and a ventral uterine precursor (VU) descendant per anterior-posterior position (except for the case of ut 4 where there is an extra VU descendant sandwiched in between) on both the left and right side of the animal. All four (or six in the case of ut4) cells then fuse to form a toroid. We speculate that the ut cells are the default state of uterine differentiation.

The structure of the uterus dorsal to the vulva is elaborated by making a hole between these epithelia, which precludes formation of a toroid in this region. Instead, the mononucleate uv2 cells contact the uterine lumen ventrally, while the du cell forms the epithelium dorsal to the lumen. Surprisingly, the DU descendants DE4 and DE5 make these cells (and the uv3 cells) although they are located ventrally as well as dorsally (see Fig. 5C). This may be caused by an absence of proximal VU-derived cells to make ventral uterine epithe-

Fig. 7. Electron micrographs, transverse sections. (A) Mid-L4, prior to AC fusion with the utse. The cell boundaries between the unfused $\mathrm{AC}$ and the vulval $\mathrm{F}$ cells are evident; the vulval E cells (E) are ventral to the vulval F cells $(\mathrm{F})$. Lateral to the AC is a utse cell with which it has not yet fused. (B) Mid-L4,

subsequent to AC fusion with the utse. Nuclei of the $\mathrm{AC}$ and of the two $\pi$ progeny to its left and right with which it has fused to make the utse. The uv1 processes extend over the dorsal surface of the F cells and contact the vulval lumen. In contrast, the apical surface of the uv 2 cells ends at the uterine lumen. du cell cytoplasm is also evident. (C) Attachment of the utse to the seam in late L4. The shape of the seam cell has become indented as a consequence of the utse attachment; the alae, a specialization of the cuticle, are lateral to the seam. The thickened uterine basal lamina (ubl) is immediately internal to the seam. The utse is internal to the basal lamina; it can be seen extending a thin process that faces both the vulval and uterine lumens. The du cell also contacts the uterine basal lamina; it curves around so that part of it is ventral and contacts uv2. uv2 faces the uterine lumen, while uv1 faces the vulval lumen, and forms junctions both with the utse and with vulF. n, nucleus; E, vulE; F, vulF; du, du cell. Bar, $2 \mu \mathrm{m}$. lium once these cells are induced as $\pi$ (see below). DE4 and DE5 must somehow be made different from their more distal sisters and cousins that contribute to the ut toroids.

\section{General issues in uterine morphogenesis}

The role of asymmetric cell divisions

There are two cell divisions during the uterine lineage that

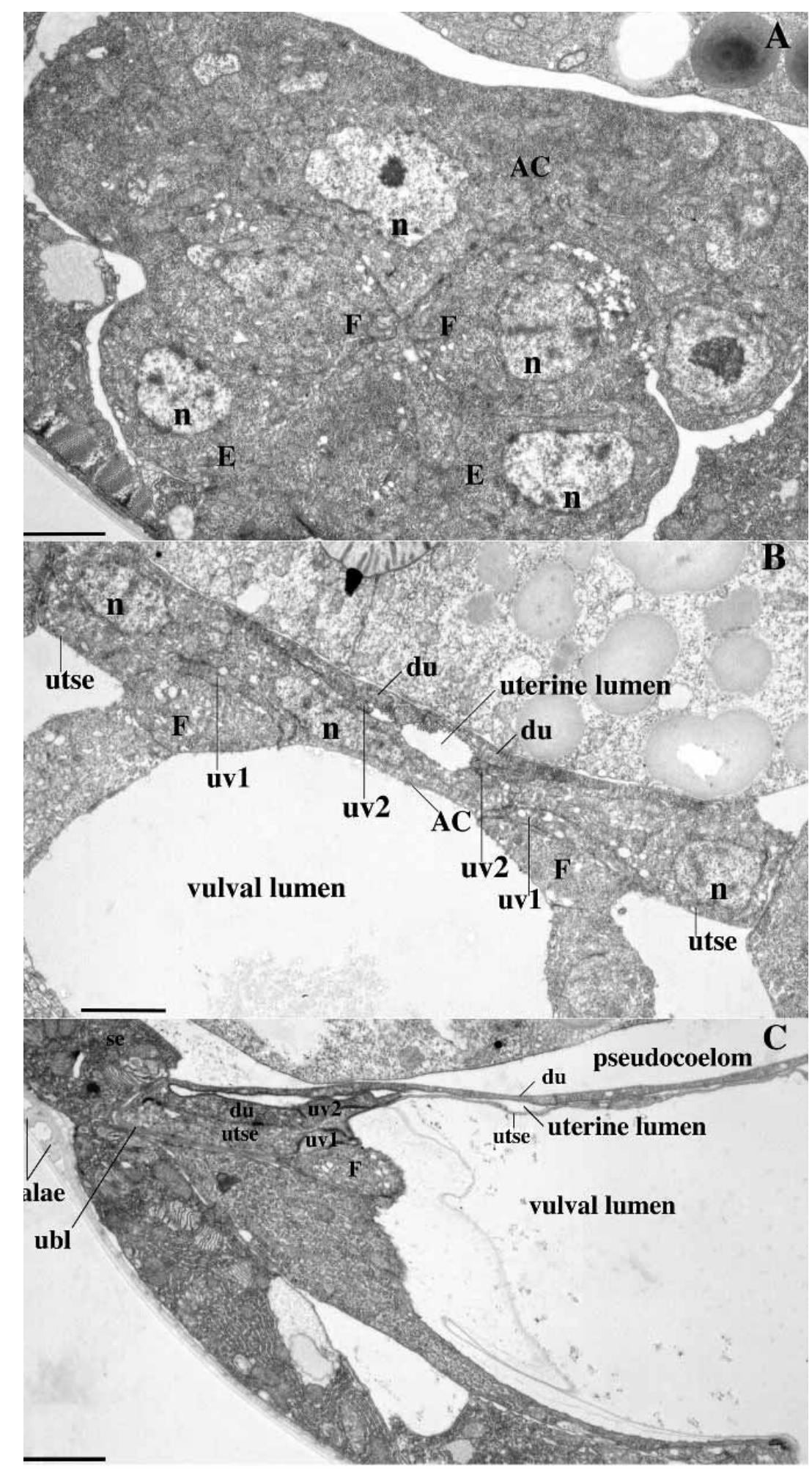


result in progeny of strikingly different size. Each DU cell daughter divides anterior-posteriorly to produce a larger proximal cell and a smaller distal cell. The proximal cells produce uterine cell types while the distal cells produce the spermathecal-uterine valve core and three cells of the spermatheca. Thus, the asymmetric cell division may contribute to the decision between uterine and spermathecal cell fates.

The $\pi$ cell division is also asymmetric, producing a larger dorsal cell and smaller ventral one. Since all dorsal daughters make utse while ventral daughters make utse and uv1 cells, the asymmetric cell division may contribute to the differentiated fate but cannot be solely responsible for determining it.

\section{Cell shape changes and migrations during uterine development}

In contrast to the situation with the ut toroids, cell migration does occur during the differentiation of certain uterine cell types. The delamination of DE4v and DE5v and the short range migrations of their progeny may be important in specifying uv2 and uv 3 fates. The utse undergoes the most dramatic changes in its position and shape during uterine morphogenesis. ACmediated induction of the $\pi$ cells that make utse occurs proximal to the vulva. However, the utse is a large cell that not only extends a process dorsal to the vulva but also extends longitudinally to ut2. This extension involves growth of a cytoplasmic process away from the site of induction and subsequent nuclear migrations.

\section{Fusions during uterine development}

Fusions are common in $C$. elegans development; about one third of the cells produced ultimately fuse (Albertson and Thomson, 1976; Podbilewicz and White, 1994; Sulston et al., 1983; White, 1988; White et al., 1976). In the C. elegans uterus, most cell types are multinucleate: the ut toroids, the uterine-spermathecal valve, the utse, and the du cell. In one reconstructed L4 animal, the ut toroids were in the process of fusing while the AC had already fused with the utse. Both fusions and lumen formation occur during mid-L4, which is thus a time of major remodeling of uterine tissue.

\section{Mechanisms generating diversity within the general uterine cell types}

As we have seen, specification of the general uterine cell types can be described by several simple steps. Yet within each general cell type, there is extensive diversity. For instance, the uv2 and uv3 cells are sisters, both are mononucleate ventral cells that are dorsal to the vulva yet do not contact it; yet their morphology by EM is strikingly different. The uterine-spermathecal valves form in a similar fashion to ut toroids although the mature valves are highly specialized. In addition, each type of ut cell may undergo a unique differentiation program. Although four (or six) cells fuse with their dorsal-ventral or left-right neighbors to form a toroid, these cells do not fuse with their anterior-posterior neighbors, and thus must have distinct properties. This further level of cell fate specification may be determined both by bona fide signaling pathways and by subtler effects caused by the position of each mature uterine cell with respect to other cells and the extracellular matrix.

\section{The uterine-vulval connection}

The AC organizes the connection of tissues

The AC first induces vulval tissue (Kimble, 1981) and then the
VU-lineage intermediate precursor cell $\pi$ fate (Newman et al., 1995). We have now demonstrated that the progeny of the $\pi$ cells become either utse or uv1 cells, the uterine cells that connect to the vulva. Thus, the AC induces production of the vulva and all uterine cells that specifically connect to it (Fig. 8). This aspect of development serves as a paradigm for how a functional passageway can be made between an internal epithelium (the uterus) and an external epithelium (the epidermis) through the action of a single cell.

The AC induces the vulva using the ligand LIN-3, and a $1^{\circ}$ vulval precursor cell, the focal point for the connection, is presumably made wherever LIN-3 concentration is highest, i.e. just ventral to the AC (Thomas et al., 1990; Hill and Sternberg, 1992; Katz et al., 1995). $\pi$ cells are induced by the AC via the receptor LIN-12 (Greenwald et al., 1983; Newman et al., 1995; Yochem et al., 1988) in an interaction probably requiring direct cell-cell contact. Thus, as with vulval induction, the appropriate uterine specialization is centered around the AC. Having a common inducing source means that specialization will be centered identically in both tissues. Yet the two epithelia do not contact one another during the time of induction; instead, the $\mathrm{AC}$ acts as a barrier between them. This may be important in allowing the cells in both tissues to differentiate independently on their own. Removal of the AC barrier is discussed below.

\section{The role of utse and uv1 cells in making the connection}

The two cell types made by $\pi$ progeny have distinct functions in making the uterine-vulval connection. The uv1 cells extend processes that directly contact and make adherens junctions with the vulval cells vulF. In contrast, the utse forms a laminar process dorsal to the vulval lumen but does not make specific junctions with the vulval cells.

In L4 stage animals, the defined dorsal-ventral sequence of cell types starting ventrally is: uv1 contacting the vulval cells and lumen and making adherens junctions to the utse; the utse facing both the vulval and uterine lumens; and uv2 facing the uterine lumen and making junctions to the utse. However, in some EM sections, the sequence is vulF, uv1, uv2, with no intervening utse. Furthermore, the utse, which functions as a hymen, is presumably broken when the first egg passes through. What then is the function of the utse dorsal to the vulva?

The utse is formed by fusion of a subset of the $\pi$ progeny with each other and with the AC. Preceding this fusion, there is thick uterine tissue dorsal to the vulva with the $\mathrm{AC}$ in its midst. Formation of the utse syncytium both removes the AC by fusing with it and replaces thick tissue with a thin process that can be broken. Furthermore, this change in the nature of uterine tissue

\section{The inductions The connection}

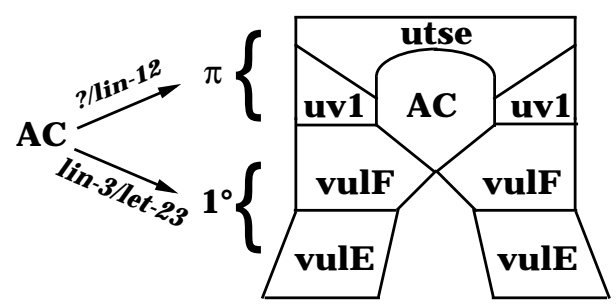

Fig. 8. Model depicting the cells making the uterine-vulval connection. The AC induces uterine $\pi$ cells which produce utse and uv1 and the vulval $1^{\circ}$ cells which produce vulF and vulE. 
dorsal to the vulva may enable this initially closed structure to open on top. Finally, specialized cells and cell junctions need to be assembled at the point where the uterine and vulval lumens meet. We speculate that it might be easier to assemble the junctional components on a planar surface. Thus, the utse appears critical to properly establish the appropriate uterine-vulval connection, although it may not be needed in the final structure.

The AC-to- $\pi$ signal followed by $\pi$ progeny engulfment of the AC: a reciprocated cell-cell interaction

Reciprocal interactions are likely important in development, for instance between ectoderm and mesoderm in vertebrate limb bud formation (reviewed by Tabin, 1995), mesoderm and endoderm in intestinal development (Roberts et al., 1995, and references therein), and between two types of mesodermal derivatives in kidney formation (reviewed by Saxen, 1987). While these examples may involve bi-directional induction, there are many ways in which two cell types can act on one another. In the $C$. elegans ventral uterus, the AC induces the $\pi$ cells. Subsequently, a subset of $\pi$ progeny fuse with the AC, causing it to change shape.

\section{The pivotal role of the $A C$ in organizing the hermaphrodite gonad}

Key events in generation of a uterine-vulval connection include: (1) induction of the vulva by the AC using LIN3/LET-23; (2) AC induction of the uterine cells that connect to the vulva using LIN-12; and (3) attachment of the two structures by formation of a thin laminar sheet (the utse syncytium) through fusion of specialized uterine cells and the AC. The AC is thus a transient cell with multiple functions that organizes the connection of two tissues into a functional structure.

This work was supported by the MRC and the Howard Hughes Medical Institute, with which P.W.S. is an investigator and A.P.N. is an associate. We thank E. Southgate and D. Kershaw for undertaking the electron microscopy, R. H. Waterston for his generous gift of the MH27 antibody, S. Crittendon for help with MH27 antibody staining, and T. Clandinin, M.-A. Felix, A. Gonzalez-Serricchio, and B. Palmer for their comments on the manuscript.

\section{REFERENCES}

Albertson, D. G. and Thomson, J. N. (1976). The pharynx of Caenorhabditis elegans. Phil. Trans. R. Soc. Lond. B. Biol. Sci. 274, 299-325.

Albertson, D. G. (1984). Formation of the first cleavage spindle in nematode embryos. Dev. Biol. 101, 61-72.

Aroian, R. V., Koga, M., Mendel, J. E., Ohshima, Y. and Sternberg, P. W. (1990). The let-23 gene necessary for Caenorhabditis elegans vulval induction encodes a tyrosine kinase of the EGF receptor subfamily. Nature 348, 693-699.

Avery, L. and Horvitz, H. R. (1987). A cell that dies during wild-type $C$. elegans development can function as a neuron in a ced-3 mutant. Cell 51, 1071-1078.

Brenner, S. (1974). The genetics of Caenorhabditis elegans. Genetics 77, 7194.

Evans, T. C., Crittendon, S. L., Kodoyianni, V. and Kimble, J. (1994). Translational control of maternal $g l p-1$ mRNA establishes an asymmetry in the C. elegans embryo. Cell 77, 183-194.

Greenwald, I. S., Sternberg, P. W. and Horvitz, H. R. (1983). The lin-12 locus specifies cell fates in Caenorhabditis elegans. Cell 34, 435-444.

Grobstein, C. (1955). Induction interaction in the development of the mouse metanephros. J. Exp. Zool. 130, 319-340.

Grobstein, C. (1956). Trans-filter induction of tubules in mouse metanephrogenic mesenchyme. Exp. Cell Res. 10, 424-440.

Henderson, S. T., Gao, D., Lambie, E. J. and Kimble, J. (1994). lag-2 may encode a signaling ligand for the GLP-1 and LIN-12 receptors of $C$. elegans. Development 120, 2913-2924.

Hill, R. J. and Sternberg, P. W. (1992). The lin-3 gene encodes an inductive signal for vulval development in C. elegans. Nature 358, 470-476.

Hirsh, D., Oppenheim, D. and Klass, M. (1976). Development of the reproductive system of Caenorhabditis elegans. Dev. Biol. 49, 200-219.

Katz, W. S., Hill, R. J., Clandinin, T. R. and Sternberg, P. W. (1995). Different levels of the $C$. elegans growth factor LIN-3 promote distinct vulval precursor fates. Cell 82, 297-307.

Kimble, J. and Hirsh, D. (1979). Post-embryonic cell lineages of the hermaphrodite and male gonads in Caenorhabditis elegans. Dev. Biol. 70, 396-417.

Kimble, J. (1981). Lineage alterations after ablation of cells in the somatic gonad of Caenorhabditis elegans. Dev. Biol. 87, 286-300.

Kimble, J. E. and White, J. G. (1981). On the control of germ cell development in Caenorhabditis elegans. Dev. Biol. 81, 208-219.

Newman, A. P., White, J. G. and Sternberg, P. W. (1995). The C. elegans lin12 gene mediates induction of ventral uterine specialization by the anchor cell. Development 121, 263-271.

Podbilewicz, B. and White, J. (1994). Cell fusions in the developing epithelia of C. elegans. Dev. Biol. 161, 408-424.

Roberts, D. J., Johnson, R. L., Burke, A. C., Nelson, C. E., Morgan, B. A. and Tabin, C. (1995). Sonic hedgehog is an endodermal signal inducing Bmp-4 and Hox genes during induction and regionalization of the chick hindgut. Development 121, 3163-3174.

Saxen, L. (1987). Organogenesis of the kidney. Cambridge University Press.

Seydoux, G. and Greenwald, I. (1989). Cell autonomy of lin-12 function in a cell fate decision in C. elegans. Cell 57, 1237-1245.

Sternberg, P. W. and Horvitz, H. R. (1986). Pattern formation during vulval development in Caenorhabditis elegans. Cell 44, 761-772.

Strome, S. (1983). Generation of asymmetry and segregation of germ-line granules in early Caenorhabditis elegans embryos. Cell 35, 15-25.

Sulston, J. and Horvitz, H. R. (1977). Postembryonic cell lineages of the nematode Caenorhabditis elegans. Dev. Biol. 56, 110-156.

Sulston, J. E. and White, J. G. (1980). Regulation and cell autonomy during postembryonic development of Caenorhabditis elegans. Dev. Biol. 78, 577 597.

Sulston, J. E., Schierenberg, E., White, J. G. and Thomson, J. N. (1983). The embryonic cell lineage of the nematode Caenorhabditis elegans. Dev. Biol. 100, 64-119.

Tabin, C. (1995). The initiation of the limb bud: growth factors, Hox genes, and retinoids. Cell 80, 671-674.

Tax, F. E., Yeargers, J. J. and Thomas, J. H. (1994). Sequence of C. elegans lag-2 reveals a cell-signalling domain shared with Delta and Serrate of Drosophila. Nature 368, 150-154.

Thomas, J. H., Stern, M. J. and Horvitz, H. R. (1990). Cell interactions coordinate the development of the C. elegans egg-laying system. Cell $\mathbf{6 2}$, 1041-1052.

Wakelom, M. O. (1988). Myoblast fusion - a mechanistic analysis. Curr. Top. Membr. Transport 32, 87-112.

Waterston, R. H. (1988). Muscle. In The Nematode Caenorhabditis elegans (ed. W. B. Wood), pp. 281-335. Cold Spring Harbor Laboratory Press, NY.

White, J. G., Southgate, E., Thomson, J. N. and Brenner, S. (1976). The structure of the ventral nerve cord of Caenorhabditis elegans. Phil. Trans. $R$. Soc. Lond. B Biol. Sci. 275, 327-348.

White, J. G., Southgate, E., Thomson, J. N. and Brenner, S. (1986). The structure of the nervous system of the nematode Caenorhabditis elegans. Phil. Trans. Roy. Soc., Lond. B. 314, 1-340.

White, J. G. (1988). The anatomy. In The Nematode Caenorhabditis elegans (ed. W. B. Wood), pp. 81-122. Cold Spring Harbor Laboratory Press, NY.

Wilkinson, H. A., Fitzgerald, K. and Greenwald, I. (1994). Reciprocal changes in expression of the receptor lin-12 and its ligand lag-2 prior to commitment in a C. elegans cell fate decision. Cell 79, 1187-1198.

Wylie, A. H., Kerr, J. F. R. and Currie, A. R. (1980). Cell death: the significance of apoptosis. Int. Rev. Cytol. 68, 251-306.

Yanagimachi, R. (1988). Sperm-egg fusion. Curr. Top. Membr. Transport 32, 3-43.

Yochem, J., Weston, K. and Greenwald, I. (1988). C. elegans lin-12 encodes a transmembrane protein similar to Drosophila Notch and yeast cell cycle gene products. Nature $\mathbf{3 3 5}, 547-550$.

(Accepted 17 July 1996) 